\title{
DIFERENTES COMPORTAMENTOS DOS USUÁRIOS DE PRODUTOS FINANCEIROS PELA INTERNET: UM ESTUDO EXPLORATÓRIO
}

\author{
DIFFERENT FINANCIAL CONSUMERS BEHAVIOR INTERNET USERS: AN EXPLORATORY STUDY
}

\author{
Favio Akiyoshi Toda \\ Pontifícia Universidade Católica do Rio de Janeiro - PUC-Rio \\ Gabriel R. D. Levrini \\ Pontifícia Universidade Católica do Rio de Janeiro - PUC-Rio
}

\begin{abstract}
RESUMO
O presente estudo tem for finalidade ampliar o entendimento do comportamento dos usuários de produtos financeiros via internet, sob uma perspectiva de Finanças Comportamentais visto que as decisões dos clientes nem sempre seguem uma decisão baseado somente em uma lógica racionalista; observa-se que em muitas decisões financeiras a lógica racional opera sobre uma superfície de um complexo conjunto de características de personalidade e atitudes dos sujeitos que exercem significativa influência sobre o comportamento em termos de realização de investimentos, compra de produtos e serviços, etc. Neste estudo, uma amostra de um publico selecionado, com elevada renda e instrução, residentes no Rio de Janeiro, Brasil e regularmente utilizando bancos por meio de ambientes virtuais, popularmente conhecidos por Internet Banking. Os resultados indicam que mesmo estando em um mesmo espaço do ambiente virtual do banco, as atitudes sobre a utilização dos produtos e serviços ofertados aos clientes divergem em função dos níveis de envolvimento que possuem para cada um dos serviços e produtos. Este trabalho foi construído com base no estudo desenvolvido por Lian e Lin (2008) de onde se adaptou para a realidade brasileira o modelo de medidas adotado pelos autores, e também serviu como orientação metodológica.
\end{abstract}

Palavras-chave: Finanças comportamentais. Comportamento do consumidor. Internet banking. Serviços on line.

\begin{abstract}
The purpose of this study, was to verify and understand the consumer financial services behaviour in the internet environment. Consumers according Behavior Finance theories not always are rational in their decisions, sometimes affected by several other complex emotional issues and this is observed in the financial services and investment decisions. In this study we made a survey using as a sample high income class citizens of Rio de Janeiro, one of the major cities in Brazil, familiar with internet home banking operations. The results showed a different behaviour among the users, close related with the intensity of their involvement with the virtual world. This study was based in Lian and Lin (2008) online products purchase behaviour adapted for the brazilian financial service market.
\end{abstract}

Keywords: Behavior finance. Consumer behavior. Internet banking. On line services. 


\section{INTRODUÇÃO}

Os bancos de varejo desde janeiro de 2010 estão aplicando um questionário de Análise de Perfil do Investidor (API) na tentativa de conhecer melhor os seus clientes e oferecer produtos mais adequados às suas necessidades. No entanto, a pesquisa apresenta contradição no comportamento, indicando que os brasileiros se acham muito mais arrojados do que realmente são, ou seja, observando as suas aplicações financeiras preferem àqueles mais conservadores, onde buscam, por exemplo, a renda fixa. Ou seja, se realmente são arrojados em sua personalidade, os investidores deveriam procurar assumir mais riscos e buscar aplicações mais rentáveis em renda variável, adequando assim a carteira ao seu perfil, ou precisam se conhecer melhor (Revista Isto E Dinheiro, 16/6/ 2010).

A pesquisa observa um perfil moderado (tolerância média ao risco) ou arrojados ( alta tolerância) mais freqüentes que os conservadores (baixa tolerância ao risco). No Banco do Brasil (BB) de uma amostra de 60 mil API's, 53\% dos clientes foram classificados como moderados e 35\% como arrojados, porém na prática, os fundos de interesse são conservadores e participam com $90 \%$ da captação do BB, que totaliza cerca de 455 bilhões. Resultados semelhantes foram encontrados no Santander e Bradesco. Segundo a ANBIMA, próximo de $12 \%$ do patrimônio dos fundos no Brasil está em carteiras mais arrojadas, sendo que a nível mundial este número alcança cerca de $40 \%$. Os bancos visam estimular a migração dos clientes para um maior interesse aos fundos de risco, diante dos atuais patamares baixos da taxas de juros quando comparados com as taxas históricas, e com a estabilidade econômica conseguida ao longo dos últimos anos.

Por outro lado, se há um trabalho de convencimento por parte dos bancos junto aos clientes em mudar os seus comportamentos com relação as aplicação em fundos de investimentos, existe também a preocupação em facilitar o acesso dos clientes aos bancos para estimulá-los para que cada vez mais façam investimentos e obtenham créditos para o crescimento dos negócios bancários. 0 acesso a novas formas de tecnologia bancária parece ser um objetivo a superar na prática diante da atual atitude de desconfiança dos clientes junto ao canal por Internet Banking, porém, a capacidade de cada pessoa em lidar com tecnologia e a sua preocupação com a segurança e o trânsito das informações na internet são fatores que trabalham de forma a desestimular esta adoção de modo geral no comércio eletrônico (Lian e Lin, 2008).

Neste novo ambiente empresarial, o setor bancário continua sendo um dos que mais investem em Tecnologia de Informação (TI), tendo a oferta de seus produtos e serviços fundamentalmente apoiados nessa tecnologia. Esse setor participa ativamente nas operações e nos processos dos demais setores, sendo também influenciado pelas transformações externas. Assim, sejam pelo seu novo ambiente ou por mudanças nos demais setores, o setor bancário é um dos mais afetados pela nova realidade de mercado. Essa situação tem exigido dos bancos um grande esforço para a assimilação e a utilização das tecnologias de informação referentes ao Comércio Eletrônico (CE, isto é, as transações de compra e venda e a utilização de serviços pela internet) na sua operacionalização e adequação nas estratégias competitivas. A multimídia interativa e a Internet estão possibilitando uma nova economia digital: os indivíduos e empresas criam riqueza, aplicando conhecimento e esforço às áreas de produção e serviços. Segundo Tapscott (1997), na fronteira digital dessa economia, estão mudando os participantes, a dinâmica, as regras, as exigências de sobrevivência e o sucesso. A despeito dessas duas forças, pode-se observar que o comércio eletrônico cresce (FolhaOnline, 2009) e, cada vez mais, torna-se um importante canal para a venda de serviços. Essas inovações no 
campo da tecnologia da informação têm causado uma reviravolta na forma de atuar das organizações, que devem preparar-se para se ajustar à realidade emergente e reinventar seus negócios com base na world wide web (Donovan, 1997). Por outro lado é preciso se preocupar- em entender os clientes, onde o comportamento do consumidor é influenciado por fatores culturais, sociais, pessoais e psicológicos, e dentre estes o mais importante são os fatores culturais segundo Kotler (2000). Outros autores observam que são as características pessoais de personalidade que são os principais determinantes sobre o comportamento online do consumidor (Amichai-Hamburger, 2002; Hills e Argyle, 2003).

Em uma pesquisa conduzida por Funfgeld e Wang (2009) na Suiça, os autores encontraram cinco agrupamentos diferentes de investidores em função de suas atitudes e práticas comportamentais relativo a assuntos financeiros, além de suas características sóciodemográficas (gênero, idade e nível de instrução). 0 estudo possui implicações gerenciais que permitem que as instituições financeiras possam desenvolver ações mais adequadas para atender as necessidades dos seus clientes para o fomento dos negócios com base em suas características. Os investidores foram agrupados em: (1) Consumidores racionais (forte envolvimento com questões financeiras e econômicas, onde o dinheiro não possuiu um fim sem si mesmo, mas uma ferramenta que precisa ser manuseada de forma inteligente, (2) Consumidores míopes (embora aparentem uma tranqüilidade com questões financeiras e econômicas, este agrupamento se preocupam menos com questões relacionadas à proteção ou formação de poupança em relação aos consumidores racionais), (c) Poupadores ansiosos (possuem significativa preocupação com a formação de uma proteção financeira, e não se sentem confortáveis sobre assuntos financeiros), (d) Seguidores limitados (clientes que não se preocupam com questões econômicas e nem se sentem desconfortáveis ou ansiosos sobre terminologias financeiras, e possuem um estilo espontâneo e intuitivo em suas decisões), e por fim os (e) gastadores ansiosos (possuem um conflito interno, pois além de gastarem com compras para reduzir suas frustrações, se preocupam em buscar uma proteção financeira).

Lian e Lin (2008) estudaram as influências que diferentes características dos produtos podem exercer sobre o comportamento dos consumidores on-line, pois até então, muitos estudos estavam focados sobre as características dos clientes sobre um tipo de produto em específico ou produtos similares. Os autores mencionam em estudos anteriores de outros autores é que os principais fatores que determinam a propensão do consumidor em aceitar e efetuar uma compra online foram as características pessoais do consumidor, valores pessoais percebidos, desenho do site, e características do próprio produto.

No sentido de ampliar o conhecimento sobre os clientes de produtos financeiros por meio da internet, o presente estudo teve o objetivo de avaliar se os serviços financeiros ofertados pelos bancos dentro de seus ambientes virtuais apresentam também diferenças em termos de aceitação em função não apenas das características dos clientes, mas também das características dos serviços financeiros em si, buscando como orientação metodológica o trabalho de Lian e Lin, citado acima. Tal questionamento pode apresentar implicações gerenciais importantes porque coloca em evidência de que além das características pessoais em termos de atitudes dos investidores, os tipos de produtos e serviços financeiros também repercutem em suas aceitações. Neste caso, as instituições financeiras podem ter que rever seus modos de atuação junto aos investidores para buscar adequar os seus discursos sobre os produtos e serviços às necessidades e particularidades dos clientes. Como mostram estudos na área de Finanças Comportamentais, as decisões dos investidores e suas práticas não são 
baseadas exclusivamente na racionalidade.

\section{REVISÃO DA LITERATURA}

\section{A REVOLUÇÃO DA INTERNET E O SETOR BANCÁRIO}

O fenômeno da Internet em nível comercial desencadeou uma abrupta expansão tecnológica e o aperfeiçoamento das transmissões de dados e do compartilhamento de informações via computadores, o aparecimento de novas formas de comercialização de produtos e serviços, dando início ao que se denominou de Internet commerce, comércio pela Internet, além de outras denominações (ecommerce, e-business, digital markets etc). Observa-se que este tema vem sendo cada vez mais enfocado, tanto na literatura de negócios quanto no âmbito acadêmico, dada a sua importância no contexto atual de um mercado cada vez mais exigente e competitivo (Schwartz, 1997; Albertin, 1998; Mougayar, 1998; Downes e Mui, 1998; Seybold, 1998).

Esta dimensão é impulsionada pela conectividade, oportunidade sem paralelo para a comunicação, colaboração, compartilhamento de recursos e acesso à informação e à quebra das barreiras de distância e tempo. Para Vassos (1997) o rápido avanço do poder da computação e a abertura comercial da Internet trouxeram oportunidades tanto para as pequenas como para as grandes empresas. O mercado já começava a exigir que as organizações procurassem cada vez mais a diferenciação e a inovação. 0 compartilhamento de informações, não só local, mas principalmente de forma global ou mundial, torna-se imprescindível para a sobrevivência num mercado muito mais competitivo que o cenário anterior. Atualmente, a maioria das estratégias de comércio eletrônico estão baseadas em soluções tecnológicas de Internet ou Internet commerce, definido como "a compra e a venda de informações, produtos e serviços, ou qualquer negócio transacionado entre duas empresas ou entre uma empresa e seus clientes (Mougayar, 1998).

Muitos bancos têm direcionado suas estratégias para aumentar a satisfação e a lealdade de seus clientes melhorando a qualidade dos serviços (Levesque e MCDougall, 1996). 0 principal argumento é que clientes satisfeitos tendem a permanecer clientes, continuando seu relacionamento comercial no futuro. Mais importante ainda é que o custo de manter clientes existentes é menor do que o custo de conquistar novos. Ademais, ao aumentar a satisfação do cliente, este pode aumentar a sua parcela de negócios, fazendo mais transações e investimentos e, assim, elevar as receitas da empresa e a rentabilidade a longo prazo (Heskett, Sasser e Schlesinger, 1997; Jones e Taylor,2007). Com tanta similaridade entre os produtos e serviços ofertados pelos bancos, o diferencial para o cliente passa a ser a qualidade com que os serviços são prestados, já que estes se assemelham tanto, que é possível, de forma geral, tratar o mercado de varejo dos bancos como um mercado de commodities (Campello e Costa Neto, 2003). Para que a diferenciação não conduza a um desperdício de recursos, o desafio dos gerentes é focar nas atividades que realmente atendam ou excedam as expectativas dos clientes. Para isso, é importante conhecer quais fatores são determinantes para os clientes quando avaliam os serviços oferecidos pelos bancos. 0 oferecimento do serviço correto é um fator alinhado com o objetivo de aumentar as taxas de retenção e, conseqüentemente, os lucros da empresa. (Levesque e MCDougall, 1996). Ademais, é importante que a atenção à diferenciação também seja voltada para a base de clientes, uma vez que segmentos específicos, atrelados a variáveis ligadas à freqüência de uso ou à preferência por determinados canais de atendimento bancário, podem ser mostrar mais ou menos receptivos a determinadas ações e estratégicas de marketing ou, ainda, apresentar diferentes níveis de 
satisfação com relação ao serviço oferecido (Kaufmann e Marchetti, 2008).

No caso das transações bancárias, com base em Tecnologia da Informação (TI), os sistemas eletrônicos de padronização de documentos pretendem melhorar a eficácia, eliminar o alto custo de tarefas rotineiras nas agências. Pela adição de valor nos processos realizados por grupos inter organizacionais, permitem que os computadores troquem informações entre si, dispensando a digitação e a manipulação de dados, tornando mais segura e rápida à transação. Via integração digital, as redes armazenam, atualizam e distribuem documentos digitais. A segurança do acesso às informações pode ser feita, por exemplo, por meio da autenticação por assinatura eletrônica, uma senha que o usuário digita na página do banco, sem necessidade de carimbo, autenticação mecânica e/ou assinatura de papéis, devido aos softwares de criptografia. Realizar transações bancárias é apenas mais um tipo de transação no universo quase infinito de possibilidades de fazer negócios pela Web. Projeções da empresa de pesquisas Forrester Research indicam que os negócios online deverão chegar a US\$1,3 trilhões em 2003. Reforçando essa previsão, uma pesquisa da Ziff-Davis Internet Trak aponta que a compra de bens e serviços online vai movimentar 17 bilhões de transações por ano até 2005 (Sun Network, 1999). 0 que ocorre no Brasil tende a ser um reflexo do que acontece em todo o mundo.

A TI é considerada fundamental para o setor bancário, tanto em nível operacional como estratégico. As razões para essa importância residem nas características do setor. Conforme definido por Crane e Bodie (1996), a indústria de serviços financeiros está se transformando de uma maneira imprevisível e, às vezes, contraditória sendo que uma das forças que têm acelerado essas mudanças é a nova tecnologia de informação. A TI é vista como uma das maiores e mais poderosas influências a serem consideradas no planejamento das instituições financeiras. Segundo Baldwin (1991), o sistema bancário, sem dúvida, está passando por uma reestruturação radical. As diretrizes fundamentais da mudança são tecnológicas e irreversíveis. As modernas tecnologias de informação, comunicação e avaliação permitem uma qualidade mais alta em vários aspectos bancários. Conforme observado por Albertin (1993), no estudo de fatores críticos de sucesso da administração de TI do setor bancário nacional, as maiores instituições bancárias brasileiras têm utilizado largamente a TI para interligar todas as suas agências em nível nacional, processar um número muito grande de transações e atender a uma quantidade de clientes dentro e fora das agências de forma rápida, segura e, muitas vezes, personalizada. A tecnologia está permitindo o desenvolvimento de novos produtos e serviços e mudando a interação entre os bancos e os consumidores de varejo.

A chave para o sucesso do sistema de Comércio Eletrônico (CE) para os clientes certamente é uma grande adoção desses tipos de tecnologias por parte deles. O relacionamento com clientes e fornecedores tende a ser significativamente alterado com a adoção intensa do CE. Os motivos são variados: eliminação de intermediários, diminuição da interação face a face, integração eletrônica disponível e facilmente acessível, maior informação sobre o cliente,,entre outros no entanto o comprometimento organizacional é imprescindível para a utilização com sucesso de um sistema de CE. Esse comprometimento inclui significativos investimentos para criação e manutenção dos sistemas, administração de mudanças organizacionais profundas, gerenciamento de riscos, etc. Uma fonte potencial de problemas é a preocupação dos clientes com privacidade e segurança, o que poderia levar a uma forte reação contra os fornecedores que utilizam tais sistemas ou simplesmente a não utilização desses sistemas por parte dos clientes (Albertin 1998). 
As transações eletrônicas de negócio somente podem ter sucesso se as trocas financeiras entre compradores e vendedores puderem acontecer em um ambiente simples, universalmente aceito, seguro e barato. Para os bancos, a TI/CE representa uma poderosa e promissora arma competitiva que deve continuar a receber significativos investimentos, devendo contribuir ara a redução de custo, para a flexibilidade e a agilidade em outras áreas, aumentando, assim, o poder competitivo dos bancos. Argumenta-se que a utilização de TI, no princípio, foi justificada pela necessidade de atender a um número bastante significativo de clientes e de produtos e serviços e pela necessidade de redução de custo ( Albertin, 1998). Os bancos têm efetivado a integração eletrônica, desde a utilização de TI tradicional até a atual utilização de TI/CE, buscando redução de custo, melhoria de processo, novos papéis nos processos de negócio e exploração de novas oportunidades.

\section{O CLIENTE DOS SERVIÇOS BANCÁRIOS}

O setor bancário é um exemplo clássico de atividade onde satisfação do cliente e recompra não são positivamente relacionados. Parece que satisfação do cliente não é necessária para garantir a lealdade, traduzida na forma de compra repetida ou na continuidade dos negócios. Bennett e Rundle- Thiele (2004) citam pesquisa realizada pela Australian Consumers Association, que mostra que $70 \%$ dos clientes de bancos são leais atitudinais: ou seja, eles pretendem continuar usando seu banco atual apesar de estarem insatisfeitos, sugerindo que a natureza da relação entre satisfação e lealdade é complexa. Acreditar que clientes satisfeitos são, obrigatoriamente, leais pode ser um engano. Em pesquisa realizada por Reichheld (1993), descobriu-se que entre $65 \%$ a $85 \%$ dos clientes que trocaram de fornecedor disseram estar satisfeitos com o fornecedor antigo. Entretanto, mesmo que a satisfação possa não se configurar como um elemento central da lealdade, após a lealdade ter sido estabelecido, é difícil que a sua manutenção se desenvolva sem a satisfação (Oliver, 1999). 0 gerenciamento das reclamações deve ser visto como uma ferramenta eficaz tanto para a retenção como para a lealdade de clientes (Milan e Trez, 2005). Ações corretivas ou mudanças em processos voltados para o aumento do nível de satisfação dos clientes deveriam diminuir a incidência de queixas (Anderson, 1998) e, conseqüentemente, um incremento no nível de satisfação possibilitaria um aumento na retenção e lealdade dos clientes (Reichheld e Sasser, 1990; Hallowell, 1996).

A automação dos serviços bancários se tornou um fator crítico no processo de tentar atingir eficácia com menor custo, o que pode ser usado como uma arma competitiva estratégica no mercado de serviços financeiros. A conveniência proporcionada pelos caixas eletrônicos tem desempenhado um papel importante e pioneiro no avanço da transformação tecnológica no cenário bancário. Em sua pesquisa, Goode e Moutinho (1995) relatam evidências encontradas na literatura que confirmam que altos níveis de satisfação de clientes de bancos estão diretamente relacionados à localização e à acessibilidade dos caixas eletrônicos, ao passo que equipamentos fora de serviço (problemas de conexão, vandalismo, etc.) eram considerados pelos clientes como uma importante fonte de insatisfação com os serviços bancários.

Kaufmann e Marchetti (2008) realizaram um estudo para verificar o efeito da utilização dos diversos canais de atendimento bancário na satisfação, valor percebido, confiança e lealdade do cliente, por meio de uma survey com 197 clientes de vários bancos. Os autores constataram que a utilização de determinados canais tem relação com o grau de satisfação do cliente com o banco, mas não com a sua lealdade. 0 estudo identificou ainda, através das análises de agrupamento, a existência de três perfis típicos de usuários segundo preferência de uso dos canais de atendimento bancário: os usuários de agência, os usuários de caixas 
eletrônicos e os usuários de Internet.

\section{FATORES QUE DETERMINAM A PROPENSÃO A REALIZAR COMPRAS DE PRODUTOS E SERVIÇOS ONLINE (LIAN E LIN, 2008)}

Lian e Lin descreveram os principais fatores que determinam a propensão do consumidor em aceitar e efetuar uma compra online até então encontrados em suas revisões bibliográficas: características pessoais do consumidor, valores pessoais percebidos, desenho do site e o produto propriamente dito. Associadas ao primeiro fator, ligado às características do consumidor, estão os traços pessoais, a sua capacidade pessoal de lidar com as várias tarefas e problemas, o seu perfil demográfico, e a sua aceitação de novas tecnologias e aplicativos de TI. O segundo fator engloba os chamados valores percebidos na transação, e as variáveis a ele associadas incluem o risco percebido, a conveniência percebida, sua percepção da qualidade do site e os benefícios aparentes a este consumidor. 0 terceiro fator, ligado ao desenho do site, inclui considerações sobre a segurança da transação e a privacidade das informações envolvidas na compra, em si, efetuada por meio deste. Finalmente, o quarto fator está ligado às características do próprio produto, e que são determinantes do sucesso da transação via internet (O'Cass e Fenech, 2003; Eastin, 2002, RSA 2006; Ranganathan e Grandon, 2002; Bhatnager, Misra e Rao, 2000).

Baseados na revisão bibliográfica, Lian e Lin construíram um modelo onde propuseram cinco construtos como críticos na determinação da atitude de um consumidor para com a compra online, e construíram seis hipóteses que foram testadas empiricamente na dimensão de quatro produtos e serviços diferentes (livros, vídeo-game, notícias online e jogos para computador, resumidos na figura 1 abaixo. Assim, o modelo buscou estudar as seguintes relações para avaliar os efeitos de diferentes produtos e serviços:

H1: altos níveis de originalidade pessoam em TI (PIIT) afetam positivamente atitudes para com compras online. Originalidade Pessoal em Tecnologia de Informação serve para traduzir a capacidade de uma pessoa em aceitar uma inovação, descrita como "o grau com que um indivíduo adota novas idéias mais cedo do que os demais" (Rogers (1995) apud Lian e Lin (2008)).

H2: altos níveis de auto-eficácia afetam positivamente atitudes para com compras online. Auto-eficácia na Internet procura traduzir a crença que um indivíduo tem na sua capacidade de dominar e usar corretamente as ferramentas de internet.

H3: altos níveis de percepção de segurança afetam positivamente atitudes para com compras online. Percepção de Segurança na Internet indica com que intensidade um indivíduo entende que as transações online são seguras, visto que elas não são feitas frente a frente.

H4: altos níveis de preocupação com a privacidade afetam negativamente atitudes para com compras online Preocupações com Privacidade procura capturar a intensidade com que um indivíduo se preocupa com a privacidade que envolve as informações trocadas e que são transferidas durante uma compra online.

H5: altos níveis de envolvimento com o produto afetam positivamente atitudes para com compras online. Envolvimento com o produto também chamada de envolvimento pessoal, tem a definição de ser "a motivação individual em relação a um objeto onde este estado de motivacional é ativado pela relevância outorgada ao objeto em questão" (Koufaris (2002) apud Lian e Lin (2008)). 
H6: Diferentes produtos e serviços afetam as relações entre características do consumidor e atitudes em relação a compras online. Diferentes Produtos e Serviços procura determinar contextos diferentes para a observação das influências das demais variáveis, dessa forma eliminado o efeito de produto, ou seja, controlando para diferenciações destes.

Figura 1 - Modelo de Pesquisa

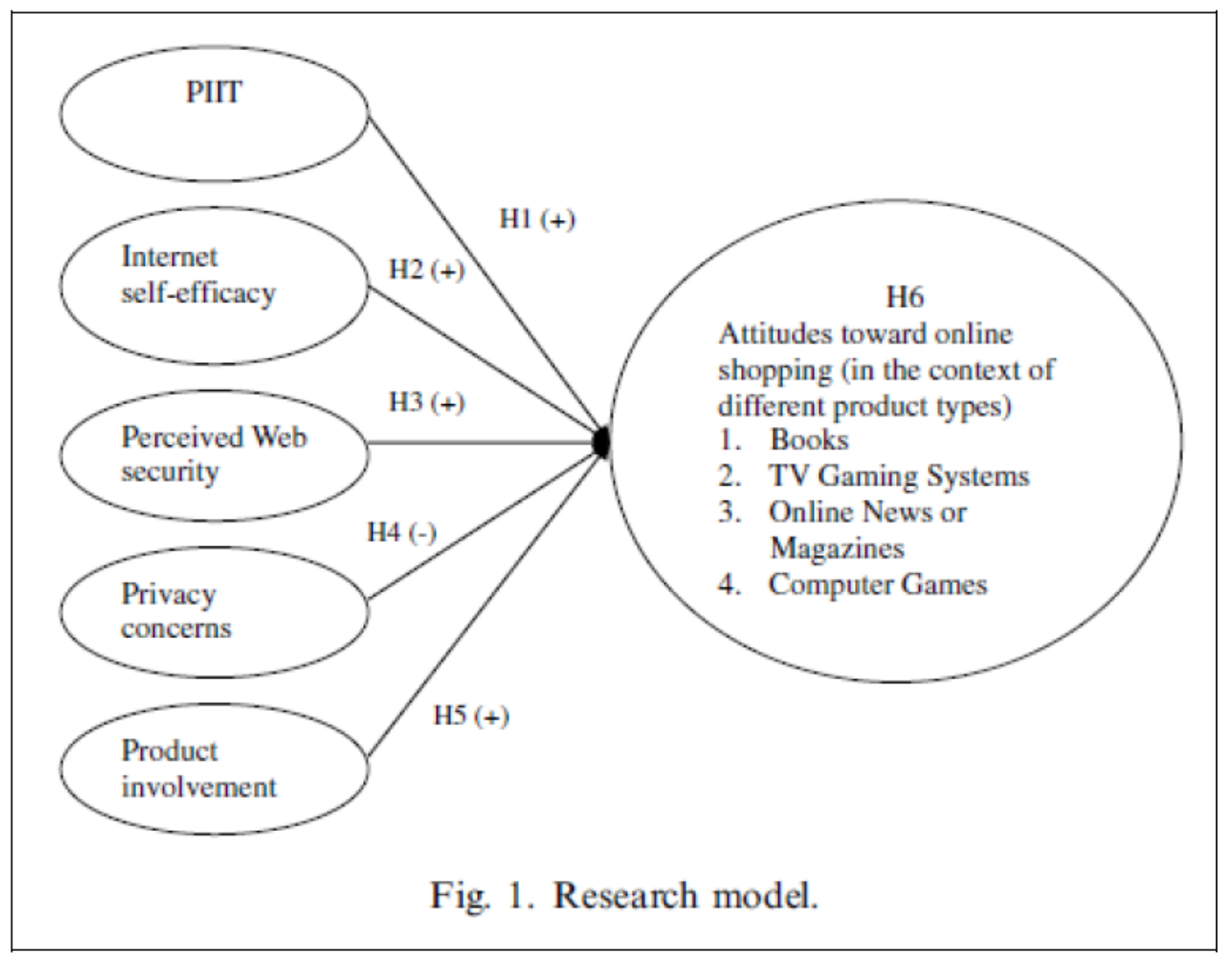

Fonte: Lian e Lin, 2008.

Por meio da aplicação da técnica de análise fatorial e regressões lineares múltipla, os autores puderam verificar que o trabalho é consistente com estudos anteriores que identificavam as características dos clientes como influenciadores na aceitação de compras online; entretanto, o estudo permitiu também verificar que tais relacionamentos são afetados pelos diferentes produtos.

\section{METODOLOGIA}

As entrevistas foram realizadas ao longo do mês de junho de 2010 através de um questionário estruturado e adaptado da versão desenvolvida por Lian e Lin (2008), onde se buscou seguir a mesma orientação metodológica tanto em termos conceituais, práticos e analíticos encontrados nos artigos dos autores acima. Porém a incompreensão de algumas partes do trabalho, e naturalmente alguns resultados muito diferentes fez com que os autores deste artigo buscassem outros tratamentos. A construção do questionário destes autores segue uma justificativa encontrada acima na revisão bibliográfica, e a versão adaptada encontra-se no anexo deste trabalho. Os construtos relativos à Originalidade Pessoal em Tecnologia de Informação foram mensurados com as perguntas P3_a até P3_d, Auto-eficácia na Internet com as perguntas P3_e até a pergunta P3_h, Percepção de Segurança na Internet com as perguntas P3_i até P3_k, Preocupações com Privacidade com as perguntas P3_L até a P3_z, Envolvimento 
com o produto com as perguntas que constam no bloco P4, e atitudes em relação a compras aos produtos e serviços financeiros com o bloco de perguntas P5. Os produtos de interesse para a pesquisa foram consulta ao saldo/ extrato, transferência entre contas-correntes, aplicações e resgates de investimentos, obtenção de crédito de médio e longo prazo (excetuando o cheque especial), e pagamento de contas.

Foram realizadas cinqüenta (50) entrevistas pelo método de amostragem por conveniência, ou seja, tem-se uma amostragem não-aleatória composta de estudantes que já possuíssem uma autonomia financeira com fonte de recursos próprios por meio de trabalho, ou em caso contrário, com um dos pais de alunos da mesma instituição de ensino - de grande prestígio nacional e localizado em uma das áreas mais nobres da capital de uma das maiores cidades do país. Os autores acreditavam que esperados elevados níveis de renda dos participantes, produzindo uma amostra homogênea nesta característica, poderia fazer com que fosse eliminada qualquer influência da disponibilidade financeira dos respondentes com relação ao uso dos serviços oferecidos pelo internet banking. Em razão de se tratar de uma amostra do tipo não-aleatória, os resultados e conclusões que se baseiam desta pesquisa quantitativa precisam ser avaliados com cuidados, pois do ponto de vista teórico não se permite generalizações dos resultados para a população.

\section{RESULTADOS}

$\mathrm{Na}$ amostra obtida, verifica-se que os entrevistados possuem em média 41,6 anos, com desvio padrão de 13,7 anos. Em termos de gênero, 55,3\% da amostra são compostas de mulheres. E $91,7 \%$ possuem no mínimo o curso superior. E 76,5\% dos respondentes que informaram as suas rendas familiares, possuem rendas acima cinco mil reais mensais. Os resultados foram analisados separadamente para cada um dos construtos apresentados na Tabela 1 e sua análise foi feita através de análise fatorial pelo método das Componentes Principais, adotando rotação VARIMAX quando foi possível de ser executado. Alguns itens foram eliminados na análise fatorial relacionado aos construtos quando o valor correspondente do MAS não alcançou o valor de 0,5, sugeridos pela literatura estatística (Hair, Tatham, Anderson, e Black, 1998) e optando-se como método de extração dos fatores, o critério pelo auto-valor acima de 1. Como se trata de um estudo exploratório, e uma amostra do tipo não-aleatório que não permite generalizações e uma quantidade de entrevistas pequena, os autores preferiram não eliminar variáveis com cargas fatoriais abaixo de 0,5 adotados por Lian e Lin (2008) com fim de captar a maior variância possível dos itens.

De acordo com a tabela 1 abaixo, verifica-se utilizando o alfa de crombach como simples medida de confiabilidade dos construtos, resultados satisfatórios excetuando a variável latente AEI (Auto-eficácia na Internet), porém muito próximo do aceitável de 0,6 para um estudo exploratório. (Hair, Tatham, Anderson, e Black, 1998) 
Tabela 1: Alfa de Cronbach dos construtos

\begin{tabular}{|c|c|c|c|c|c|c|c|c|c|}
\hline \multicolumn{5}{|c|}{ Variáveis Dependentes } & \multicolumn{5}{|c|}{ Atitude para o Serviço } \\
\hline variáveis & OPTI & AEI & PSI & PP & $\begin{array}{c}\text { Consulta a } \\
\text { saldo/ } \\
\text { extrato }\end{array}$ & $\begin{array}{c}\text { Transferê } \\
\text { ncia de } \\
\text { valores } \\
\text { entre } \\
\text { contas- } \\
\text { correntes }\end{array}$ & \begin{tabular}{|c|} 
Aplicações \\
ou retiradas \\
$\mathrm{em}$ \\
investimento \\
$\mathrm{s}$
\end{tabular} & $\begin{array}{c}\text { Obtenção } \\
\text { de crédito } \\
\text { de médio e } \\
\text { longo } \\
\text { prazo }\end{array}$ & \begin{tabular}{|l} 
Pagament \\
o de contas
\end{tabular} \\
\hline$n^{\circ}$ itens & 4 & 3 & 3 & 14 & 5 & 5 & 5 & 5 & 5 \\
\hline$\alpha$ & 0,672 & 0,589 & 0,788 & 0,883 & 0,877 & 0,902 & 0,896 & 0,920 & 0,880 \\
\hline
\end{tabular}

\begin{tabular}{|c|c|c|c|c|}
\hline \multicolumn{5}{|c|}{ Envolvimento com o serviço } \\
\hline $\begin{array}{c}\text { Consulta a } \\
\text { saldo/ } \\
\text { extrato }\end{array}$ & $\begin{array}{c}\text { Transferência } \\
\text { de valores } \\
\text { entre contas- } \\
\text { correntes }\end{array}$ & $\begin{array}{c}\text { Aplicações } \\
\text { ou retiradas } \\
\text { em } \\
\text { investimento }\end{array}$ & $\begin{array}{c}\text { Obtenção } \\
\text { de crédito } \\
\text { de médio e } \\
\text { longo }\end{array}$ & $\begin{array}{c}\text { Pagamento } \\
\text { de contas }\end{array}$ \\
8 & 4 & 9 & $\begin{array}{c}\text { prazo } \\
10\end{array}$ & 10 \\
0,885 & 0,822 & 0,721 & 0,726 & 0,683 \\
\hline
\end{tabular}

Ao fim das análises fatoriais sobre cada construto, verificaram-se as seguintes quantidades de fatores para cada construto, de acordo com a tabela 2:

Tabela 2: Alfa de Cronbach dos Construtos

\begin{tabular}{|c|c|c|c|}
\hline Construtos & Definição & Questões & Fatores \\
\hline $\begin{array}{l}\text { OPTI (Originalidade Pessoal em Tecnologia de } \\
\text { Informação) }\end{array}$ & $\begin{array}{l}\text { A intensidade com que um individuo adota/experimenta } \\
\text { novas formas de Tecnologia de Informação mais cedo }\end{array}$ & 4 & 1 \\
\hline AEI (Auto-eficácia na Internet) & $\begin{array}{l}\text { A crença de cada individuo em sua capacidade de } \\
\text { dominar e usar corretamente as ferramentas de internet. }\end{array}$ & 4 & 1 \\
\hline PSI (Percepção De Segurança na Internet) & $\begin{array}{l}\text { A intensidade com que um individuo entende que as } \\
\text { transações online são seguras. }\end{array}$ & 3 & 1 \\
\hline PP (Preocupação com Privacidade) & $\begin{array}{l}\text { O grau de preocupação de um individuo com a } \\
\text { privacidade das informações enviadas durante uma } \\
\text { compra online. }\end{array}$ & 15 & 4 \\
\hline \multirow[t]{6}{*}{ EP (Envolvimento com Produto) } & $\begin{array}{l}\text { A importância que um individuo confere a um } \\
\text { determinado produto ou serviço. }\end{array}$ & & \\
\hline & consulta ao saldo e extrato & 8 & 2 \\
\hline & transferência de valores entre contas correntes & 2 & 1 \\
\hline & aplicaçôes ou retirada em investimentos & 9 & 2 \\
\hline & $\begin{array}{c}\text { obtenção de crédito (empréstimo de médio e } \\
\text { longo prazo, retira-se do caso o cheque especial) }\end{array}$ & 10 & 3 \\
\hline & pagamento de conta & 10 & 3 \\
\hline
\end{tabular}

Para avaliar o alinhamento do modelo teórico com os dados empíricos, foram feitas regressões lineares multivariadas, tendo a medida de Atitude para cada um dos serviços financeiros (consulta ao saldo/ extrato, transferência entre contas, aplicações e resgates de investimentos, obtenção de crédito, e pagamento de contas) como variável dependente e uma combinação linear dos fatores extraídos para as características dos consumidores descritos acima como variáveis independentes: 
A fórmula da equação ajustada pode ser vista na equação abaixo:

Atitude para o serviço $\mathrm{Yi}=\alpha+\beta_{1}$ OPTI $+\beta_{2} \mathrm{AEI}+\beta_{3} \mathrm{PSI}+\left(\beta_{41} \mathrm{PP} 1+\beta_{42} \mathrm{PP} 2+\right.$

$$
\left.\beta_{43} \mathrm{PP} 3+\beta_{44} \text { PP4 }\right)+\left(\Sigma \beta_{51 \_1.55_{-} 3} \text { EP Yi }\right)
$$

onde Yi é o tipo de serviço e os ß’s de EP estão em função da quantidade fatores presentes em cada tipo de serviço

Assim, foram feitas cinco regressões independentes, uma para cada um dos cinco serviços oferecidos pelo internet banking. Os resumos dos resultados para as regressões efetuadas encontram-se na Tabela 3.

Tabela 3: Análises da qualidade das Regressões

\begin{tabular}{|c|r|r|r|r|r|}
\hline Produtos $\rightarrow$ & $\begin{array}{c}\text { Consulta a } \\
\text { saldo/ extrato }\end{array}$ & $\begin{array}{c}\text { Transferência de } \\
\text { valores entre } \\
\text { contas-correntes }\end{array}$ & $\begin{array}{c}\text { Aplicaçoes ou } \\
\text { retiradas em } \\
\text { investimentos }\end{array}$ & $\begin{array}{c}\text { Obtenção de } \\
\text { crédito de } \\
\text { médio e longo } \\
\text { prazo }\end{array}$ & $\begin{array}{c}\text { Pagamento de } \\
\text { contas }\end{array}$ \\
\hline Estatísticas $\downarrow$ & 0,569 & 2,007 & 1,243 & 4,224 & 0,861 \\
\hline$F$ & 0,814 & 0,700 & 0,297 & 0,001 & 0,576 \\
\hline probabilidade (p-value) & 0,114 & 0,281 & 0,219 & 0,520 & 0,181 \\
\hline $\boldsymbol{R}^{2}$ & 2,404 & 2,388 & 1,818 & 1,635 & 2,200 \\
\hline Durbin-Watson & & & & &
\end{tabular}

Entre as regressões observadas acima, verifica que a única regressão que apresenta validade é quanto à obtenção de crédito de médio e longo prazo, onde inclusive o R2 encontrado não é alto de 0,52. Como o valor de Durbin-Watson encontra-se entre 1,11 e 2,04, os resíduos não apresentam problemas de auto-correlação. Abaixo, segue os resultados para os parâmetros estimados desta única regressão válida.

Tabela 4: Estimativa dos coeficientes da regressão sobre o Serviço Crédito

\begin{tabular}{|c|c|c|c|c|c|c|c|}
\hline & $\begin{array}{l}\text { Unstan } \\
\text { Coeffi }\end{array}$ & $\begin{array}{l}\text { lardized } \\
\text { cients }\end{array}$ & $\begin{array}{c}\text { Standardiz } \\
\text { ed } \\
\text { Coefficient } \\
\text { s }\end{array}$ & & & Collinearit & atistics \\
\hline & B & Std. Error & Beta & $\mathrm{t}$ & Sig. & Tolerance & VIF \\
\hline (Constant) & $4,19 \mathrm{E}-17$ & 0,11 & & 0 & 1 & & \\
\hline OPTI & 0,198 & 0,15 & 0,198 & 1,324 & 0,193 & 0,548 & 1,824 \\
\hline AEI & $-0,287$ & 0,124 & $-0,287$ & $-2,322$ & 0,026 & 0,803 & 1,245 \\
\hline PSI & 0,042 & 0,148 & 0,042 & 0,284 & 0,778 & 0,562 & 1,779 \\
\hline PP1 & 0,035 & 0,12 & 0,035 & 0,294 & 0,77 & 0,857 & 1,167 \\
\hline PP2 & 0,038 & 0,12 & 0,038 & 0,319 & 0,751 & 0,861 & 1,162 \\
\hline PP3 & 0,075 & 0,117 & 0,075 & 0,637 & 0,528 & 0,898 & 1,114 \\
\hline PP4 & 0,204 & 0,118 & 0,204 & 1,73 & 0,092 & 0,888 & 1,126 \\
\hline EP1_CRED & $-0,41$ & 0,125 & $-0,41$ & $-3,291$ & 0,002 & 0,794 & 1,259 \\
\hline EP2_CRED & 0,21 & 0,121 & 0,21 & 1,74 & 0,09 & 0,846 & 1,182 \\
\hline EP3_CRED & $-0,227$ & 0,118 & $-0,227$ & $-1,922$ & 0,062 & 0,88 & 1,137 \\
\hline
\end{tabular}


Observando os resultados da tabela 4, verifica-se que apenas os coeficientes das variáveis dependentes AEI, EP1_CRE mostram-se significantes ao nível de 0,05. Quanto à análise dos resíduos observou-se: (a) teste de Kolmogorov-Smirnov mostra que os resíduos da regressão apresentam-se normais, (b) o exame dos VIF's acima mostram-se aceitáveis (abaixo de 10) em termos de ausência de multicolinearidade. (c) e o teste Pesaran-Pesaran realizado sobre a avaliação da presença de homecesdacidade não foi rejeitado.

\section{CONCLUSÕES E RECOMENDAÇõES}

O objetivo deste trabalho foi atingido em parte quando obteve evidência suportando a hipótese de que os níveis de envolvimento que os consumidores possuem com produtos ou serviços distintos afetam positivamente a atitude destes com relação à realização online dos serviços oferecidos. Neste caso, observou-se com relação à realização de um serviço online de obtenção de crédito de médio e longo prazo, e não se verificou em outros quatro serviços testados (consulta ao saldo/ extrato, transferências entre contas-correntes, aplicações ou resgates em investimentos e pagamento de contas). Além disto, diferentemente da teoria e do estudo Lian e Lin (2008) os resultados não evidenciaram nesta pesquisa que os construtos, exceto para a Auto-Eficácia na Internet (AEI) - embora sem forte constatação, relações que explicassem a atitude frente ao consumo on-line de serviços bancários oferecidos no meio virtual. Ou seja, a principal constatação deste artigo é que se tratando da necessidade de obtenção de crédito por parte dos clientes, esta variável se mostra como a mais significativa para explicar sobre a atitude mais positiva dos clientes com relação à realização deste serviço específico pela Internet, e em nenhum outro produto pode-se verificar a mesma resposta. Uma possível conclusão para confirmação em estudos futuros é de que a força da necessidade pelo produto é a principal variável que explicaria a atitude dos clientes perante os serviços relativos aos produtos oferecidos pelos bancos.

Para estudos posteriores, sugere-se que outras pesquisas sejam feitas na mesma linha, procurando superar as aparentes limitações deste trabalho em termos de ampliar referenciais teóricos que possam trazer outras perspectivas de explicação para a atitude ao consumo online de serviços financeiros oferecidos pelos bancos, tão como aumentar a amostra para obter maior representatividade e poder de teste. Os autores depois deste trabalho vislumbram implicações para outros estudos em outras áreas decorrentes da possibilidade de transpor os conceitos abordados neste artigo em outras áreas de Finanças, por exemplo, de que (a) as compras de terminadas ações, e outros tipos de investimentos podem estar mais associados às atitudes positivas formadas ao longo do tempo entre os investidores e corretores, do que propriamente ao desempenho objetivo das ações ao longo do tempo, ou até mesmo com relações às expectativas futuras de retorno das ações. Outro tema que pode ser estudado é de que mesmo parecendo óbvio que os tipos de investimentos realizados dependem das atitudes que os clientes possuem para cada um deles, é procurar determinar quais variáveis ou características de personalidade são determinantes para estas atitudes dos investidores para explicar as suas escolhas para os variados produtos.

\section{BIBLIOGRAFIA}

ALBERTIN, A. L. Comércio eletrônico : um estudo no setor bancário. In: XXII ENCONTRO ANUAL DA ANPAD (1998 : Foz do Iguaçu). Anais Eletrônicos... Foz do Iguaçu :ANPAD, 1998.

ANDERSON, E. W. Customer satisfaction and word-of-mouth. NQRC (National Quality ResearchCenter), The University of Michigan, July 23, 1998 
AMICHAI-HAMBURGER, Y., Internet and personality, Computers in Human Behavior, v. 18, n. 1, 2002, p. 1-10.

BENNETT, R., RUNDLE-THIELE, S. Customer Satisfaction should not be the only goal. Journal of Services Marketing, v. 18, n. 7, p. 514-523, 2004.

BHATNAGER, A, MISRA, S, RAO, H. R, "On risk, convenience, and internet shopping behavior", Communications of the ACM, v. 43, n. 11, 2000, p. 98-105.

BUTCHER, K; SPARKES, B.; O'CALLAGHAN, F. Evaluative and relational influences on service loyalty. International Journal of Service Industry Management, v. 12, 4, p.310-327, 2001.

CAMPELLO, M. L. C.; COSTA NETO, P. L. O. A qualidade como fator de competitividade dos bancos de varejo no Brasil, 2001. Disponível em http://www.simpep.feb.unesp.br/simpep2007/anais10/ana10c.htm. Acesso em 15.01.2007.

CARO, A, "Fatores Críticos no Comportamento do Consumidor Online: um Estudo Exploratório", Dissertação de Mestrado, DA-USP, 2005.

DONOVAN, J. J. The second industrial revolution: reinventing your business on the web. Englewood Cliffs, NJ : Prentice-Hall, 1997.

DOWNES, L.; MUI, C. Unleashing the killer ap: digital strategies for market dominance. Boston, MA : Harvard Business School Press, 1998.

DUFFY, J. A.; MILLER, J.; BEXLEY, J. B. Banking customers' varied reactions to service recovery strategies. International Journal of Bank Marketing. v. 24, n. 2; p. 112-132, 2006.

EASTIN, M Diffusion of E-commerce: An analysis of the adoption of four E-commerce activities, Telematics and Informatics, v. 19, n. 3, 2002, p. 251-267.

FUNFGELD, B. WANG, M. Attitudes and behaviour in everyday finance: evidence from Switzerland. International Journal of Bank Marketing. Vol. 27 No. 2, 2009. pp. 108-128

GARSON, D Scales and Standard Measures, North Carolina State University, disponível em http://www2.chass.ncsu.edu/garson/pa765/standard.htm, acesso em 12/junho/2010.

GERRARD, P.; CUNNINGHAM, J. B. DEVLIN, J. F. Why consumers are not using internet banking: a qualitative study. Journal of Services Marketing, v. 20. n. 3, p. 160-168, 2006.

GOODE, M., MOUTINHO, L. The effects of free banking on overall satisfaction: the use of automated teller machines. International Journal of Bank Marketing, v. 13 n. 4, p. 33-40, 1995.

HAIR Jr., J. F.; ANDERSON, R. E.; TATHAM, R. L.; BLACK, W. C. Multivariate data analysis. 5.ed.. Upper Saddle River: Prentice Hall, 1998.

HALLOWELL, R. The relationships of customer satisfaction, customer loyalty, and profitability: an empirical study. International Journal of Service Industry Management, v. 7, n. 4, p. 27-42, 1996.

HART, C. W. L., HESKETT, J. L., SASSER JR., E. W. The profitable art of service recovery. Harvard Business Review, v. 68, n. 4, p. 148-56, 1990. 
HAUSKNECHT, D.C. Measurement scales in customer satisfaction/dissatisfaction. Journal of Consumer Satisfaction, Dissatisfaction and Complaining Behaviour, v 3, p. 1-1,. 1990.

HESKETT, J. L.; SASSER JR., W. E; SCHLESINGER, L. A. The Service Profit Chain. The Free New York: Press, 1997.

HILLS, P. ARGYLE, M, Uses of the internet and their relationships with individual differences in personality, Computers in Human Behavior, v. 19, n. 1, p. 59-70, 2003

HIRSCHMAN, A.O. Exit, Voice and Loyalty. Cambridge, MA: Harvard University Press, 1970

JAMAL A.; NASER K. Customer satisfaction and retail banking: an assessment of some of the key antecedents of customer satisfaction in retail banking. The International Journal of Bank Marketing, v.20, n. 4, p. 146-160, 2002.

JONES, T. O.; SASSER JR., W. E. Why satisfied customers defect. Harvard Business Review, v. 73, n.6, p. 88-99, 1995.

JONES, T.; TAYLOR, S. F. The conceptual domain of service loyalty: how many dimensions? Journal of Services Marketing. v. 21, n.1, p. 36-51, 2007.

KAUFFMANN, S; MARCHETTI, R. Z. Canais de atendimento bancário e satisfação do cliente: um estudo em bancos de varejo. In: ENCONTRO DE MARKETING DA ASSOCIAÇÃO NACIONAL DOS PROGRAMAS DE PÓS-GRADUAÇÃO EM ADMINISTRAÇÃO, 3., 2008, Curitiba. Anais.. Rio de Janeiro: ANPAD, 2008.

KOTLER, P. Administração de Marketing, 10ª edição, São Paulo:Prentice Hall, 2000.

KRISHNAN, M. S.; RAMASWAMY, V.; MEYER, M. C.; DAMIEN, P. Customer satisfaction for financial services: the role of products, services, and information technology, Management Science, v.45, n. 9, p. 1194-1209, 1999.

LASSAR, W. M.; MANOLIS, C.; WINSOR, R.D. Service quality perspectives and satisfaction in private banking. International Journal of Bank Marketing, v. 18, n. 4, p. 181-199, 2000.

LEVESQUE, T; MCDOUGALL, G. H. G. Determinants of customer satisfaction in retail banking. International Journal of Bank Marketing. v.14, n.7, p.12-20, 1996.

LIAN, J.-W., LIN, T.-M., Effects of consumer characteristics on their acceptance of online shopping: Comparisons among different product types, Computers in Human Behavior, v. 24, p. 48-65, 2008.

MADILL, J. J.; FEENEY, L.; RIDING, A.; HAINES JR., G. H. Determinants of SME owners' satisfaction with their banking relationships: a Canadian study. International Journal of Bank Marketing, v. 20, n.2, p. 86-98, 2002.

MALHOTRA, N, Marketing Research, 5ª edição, New Jersey:Prentice Hall, 1997.

MEUTER, L. M.; OSTROM, A. L.; ROUNDTREE R. I.; BITNER, M. J. Self-service technologies: understanding customer satisfaction with technology-based service encounters, Journal of Marketing, v. 64, p. 50-64, 2000.

MILAN, G. S.; TREZ, G. Pesquisa de satisfação: um modelo para planos de saúde. RAE- 
eletrônica v.4, n.2, 2005.

MOUTINHO, L.; SMITH, A. Modelling bank customer satisfaction through mediation of attitudes towards human and automated banking. International Journal of Bank Marketing, v. 18, n.3, p.124- 34,2000.

MOUGAYAR, W. Opening digital markets: battle plans and business strategies for Internet commerce. 2. ed. New York : McGraw-Hilll, 1998.

O'CASS, A, FENECH, T, Web retailing adoption: Exploring the future of Internet users web retailing behavior, Journal of Retailing and Consumer Services, v. 10, N. 2, P 81-94, 2003.

OLIVEIRA, R. A. C. O Internet banking e os hábitos de uso entre os clientes pessoa física: atributos e resistências. Dissertação de Mestrado. PPGA - UFRGS, 2001.

OLIVER, R. L. Whence consumer loyalty? Journal of Marketing, v. 63, n. 4, p. 33-44, 1999

PARASURAMAN, A.; BERRY, L.L.; ZEITHAML, V.A Understanding customer expectations of service. Sloan Management Review, v. 32, n.3, p. 39-48, 1991.

PETERSON, R, BALASUBRAMANIAN, S, BRONNENBERG, B, Exploring the implications of the Internet for consumer marketing, Journal of Academy of Marketing Science, v. 25, n. 4, p. 329-346, 1997.

RAMOS A., COSTA F. , Serviços Bancários pela Internet: um Estudo de Caso Integrando a Visão de Competidores e Clientes, RAC, v. 4, n. 3, Set./Dez. 2000

RANGANATHAN, C., e GRANDON, E, An exploratory examination of factors affecting online sales, Journal of Computer Information Systems, v. 42, n. 2, Spring p. 87-93, 2002.

REICHHELD, F. F. Loyalty-based management. Harvard Business Review, v. 71, n.2, p. 64-73, 1993.

REICHHELD, F. F. The one number you need to grow. Harvard Business Review, v. 83, n. 6, 2003.

REICHHELD, F. F.; SASSER Jr., E. W. Zero defections: quality comes to service. Harvard Business Review, v. 68, n. 5, p. 105-111, 1990.

RESENDE, T, Com impulso do Ponto Frio, Pão de Açúcar quer liderança no comércio eletrônico, FolhaOnline, São Paulo, 12/nov/2009. Disponível em http://www1.folha.uol.com.br/folha/dinheiro/ult91u651487.shtml, acesso em 11/dez/2009.

ROGERS, E, Diffusion of innovations, 4ํㅗ edição, New York:The Free Press, 1995.

RSA, Consumer Testing Reinforces Need for Simplicity and Convenience in Online Security Measures, RSA Press Releases, 17/jul/2006. Disponível em http://www.rsa.com/press_release.aspx?id=70370, acesso em 12/jun/2010.

SEYBOLD, P. B. Customers.com: how to create a profitable business strategy for the Internet and beyond. New York : Radom House, 1998.

SMITH, J, MILBERG, S, BURKE, S, Information privacy: Measuring individuals' concerns about 
organizational practices, MIS Quarterly, v. 20, n. 2, , p. 167-196, 1996.

SMITH A. K.; BOLTON R. N. An experimental investigation of customer reactions to service failures: paradox or peril? Journal of Service Research, v 1, n. 1, p. 65-81, 1998.

SOUZA NETO, A. F.; FONSECA, F. R. B.;OLIVEIRA, P. A. S. Dimensões do relacionamento e variáveis demográficas: uma investigação com base nas opiniões dos clientes de um grande banco brasileiro. ENCONTRO NACIONAL DA ASSOCIAÇÃO NACIONAL DOS PROGRAMAS DE PÓS-GRADUAÇÃO EM ADMINISTRAÇÃO, 29., .. Brasília. Anais... Rio de Janeiro: ANPAD, 2005.

SOARES, R. O.; HOPPEN, N. Aspectos do uso da Internet pelas grandes empresas no Brasil : um estudo exploratório baseado em sites web. XXII ENCONTRO ANUAL DA ANPAD (1998 : Foz do Iguaçu). Anais Eletrônicos... Foz do Iguaçu :ANPAD, 1998.

SUN NETWORK. We're the dot in.com. Revista da Sun Microsystems do Brasil, v.8, n. 22, p. 22, ago. 1999.

TAPSCOTT, D. Economia digital: promessa e perigo na era da inteligência em rede. São Paulo : Makron Books, 1997.

TV SENAC-SP. Internet dos negócios. Programa Ver e Rever, 1998..

VASSOS, T. Marketing estratégico na Internet. Indianápolis : Que Corporation, 1997.

REVISTA ISTO É DINHEIRO, 16/6/2010 Ano 13 No 662, Editora Abril. 